\title{
Flowability of a mixture of two powders obtained by co-grinding, mixing and surface treatment
}

\author{
A. Kouadri-Henni, N. Azema *, A. Benhassaine \\ Laboratoire Génie Particulaire, Ecole des Mines d'Alès, 6, avenue de Clavières, 30319 Alès cedex, France
}

\begin{abstract}
Usage properties of powder mixtures like flowability and floodability depend strongly on their granular characteristics: size, shape, surface and spread of particle size. The products used are mixtures elaborated from two micron sized powders, made of synthetic minerals. Their particle size distributions are each initially mono-modal, respectively $5 \mu \mathrm{m}$ and $70 \mu \mathrm{m}$. The mixture elaboration procedure consists in making several successive combined stages of co-grinding, mixing and surface treatment by addition of surfactants. We obtain thus several mixtures $M_{i}$ having different rheological behaviours and particle size distributions-ascertained on a electron beam microscope. The results permitted in understanding the influence of the granular macrostructure and the effect of a surface treatment on a powder's rheological behaviour.
\end{abstract}

Keywords: Co-grinding; Surface treatment; Floodability; Flowability; Particle size

\section{Introduction}

The principal aim of the study is to link the parameters of a combination of co-grinding, mixing and surface treatment with the particle size distribution and floodability of the resulting powders. Floodability is defined as the study of movement of a powder when it passes from the state of repose to the state of movement or inversely-all that under stress. Floodability corresponds to the angle of difference described by Carr [1]. In fact powder solids have specific properties which control their behaviours (flowability, agglomeration...) at the moment of use (compaction, dosage, etc.) or ulterior handling (storage, transport...). These properties depend strongly on their particle size distribution: size and size spread, shape, surface. We propose to study the influence of their parameters on the rheological behaviour of powders and to connect the granular macrostructure to flowability, compressibility and floodability. The products used are mixtures made by mixing from two micron-sized powders (Aluminium Fluoride $\mathrm{P}_{1}$ and Aluminium hydroxide $\mathrm{P}_{2}$ ), made of synthetic

\footnotetext{
* Corresponding author. Tel.: +33-4-66-78-50-00; Fax: +33-4-66-7850-34
}

minerals whose general characteristics cannot be presented here because they are the object of an industrial application. Quantities used between $\mathrm{P}_{1}$ and $\mathrm{P}_{2}$ are obtained by the ratio $\mathrm{P}_{2} / \mathrm{P}_{1}=1.9$. Their particle size distributions are initially mono-modal confirmed by laser diffraction in an alcoholic medium and are respectively $5 \mu \mathrm{m}$ with a $d_{90}-d_{10}$ spread of $8 \mu \mathrm{m}$ and $70 \mu \mathrm{m}$ with $d_{90}-d_{10}$ spread of 80 $\mu \mathrm{m}$. The mixing procedure consists in making several successive combinations of co-grinding, mixing and surface treatment by addition of surfactants. These surfactants are two polyols A and B, organic molecules, in which we vary two parameters: concentration by weight-from $0 \%$ to $5 \%$ - and carbon chain length (Fig. 1). The chain of surfactant B is longer than the chain of surfactant A. They both allow modifying the chemical nature of the mineral surface [2,3]. We thus obtain several mixtures $M_{i}$ having different rheological behaviours and particle size analyses ascertained on a electron beam microscope.

\section{Procedures for obtaining powders}

The objective is to obtain from a mixture of two powders $\mathrm{P}_{1}$ and $\mathrm{P}_{2}$ having different sizes, a powder which is homogeneous in both mixing and size. The procedure 


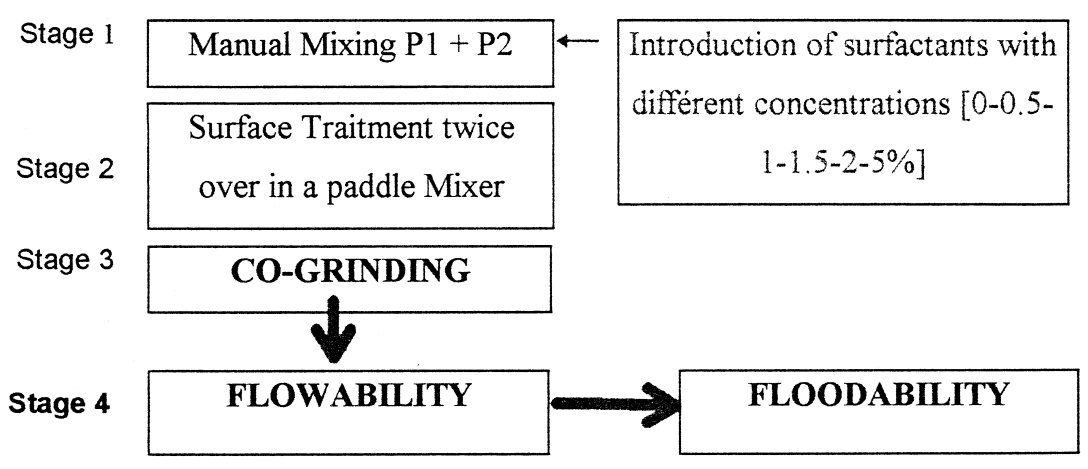

Fig. 1. Manufacturing procedure.

consists of several mixing stages followed by a co-grinding of both powder and surfactant (Fig. 1) [4]. Our objective will be to prepare homogeneous mixtures [5] and to check their granular macrostructure with the intention of improving flowability and decreasing the risks of agglomeration through addition of surfactants.

Stages 1 and 2 permit surface treatment and mixing.

To carry out stage 2, we used a 'Draiss Eirich' mixer. It allows introducing the surfactants dry and obtaining a good distribution throughout the powder and homogenisation of the mixture [6]. The different mixing stages allowed us to treat the particle surfaces using different concentrations of surfactants.

For stage 3, we used a ball-mill which had the advantages of doing the grinding and the mixing at the same, the aim being to achieve a homogeneous mixture by reduction of size. Finally, we used a sampler in order to obtain regular mixing.

\section{Experimental techniques}

The rheology of the mixtures was evaluated with a powder characteristics tester (Hosokawa) [1]:

-The compressibility is measured from apparent aerated and apparent packed densities.

-The flowability is determined by the measurement of angle of repose and angle of fall.

-The floodability is obtained from the difference between the angles of repose and of fall.

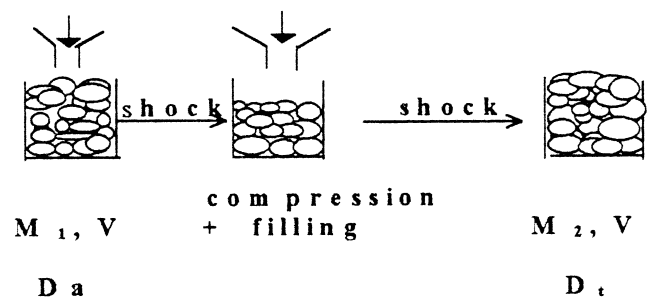

Fig. 2. Principle of measurement of compressibility (\%).

\subsection{Measurement of compressibility}

Compressibility is a function of the difference between apparent aerated density and the apparent packed density. The experiment consists in filling a recipient of volume $V$ with powder through a vibrating filter and weighing the result $M_{1}$. After that, we compact the powder by means of a compressive shock then add enough powder to restore the volume to what it was. We then weigh the result again, $M_{2}$. The apparent aerated density $D_{\mathrm{a}}$ is obtained by the ratio $M_{1} / V$. The apparent packed density $D_{\mathrm{t}}$ is $M_{2} / V$ as shown by Fig. 2.

The compressibility (\%) is obtained by following calculation:

$\left[\left(D_{\mathrm{t}}-D_{\mathrm{a}}\right) / D_{\mathrm{t}}\right] \times 100$.

\subsection{Measurement of angle of repose}

The angle of repose is the angle between the horizontal and slope of the heap. This angle is a direct indication of the potential flowability of a powder (contact and friction between particles in motion). The powder flows out from the end of a vibrating funnel [4] (Fig. 3).

\subsection{Measurement of angle of fall}

When the angle of repose of the powder becomes constant, we drop a weight on the table to one side of the heap, to give a shock to the pan. We repeat this three times. The powder heap collapses, yielding a smaller angle

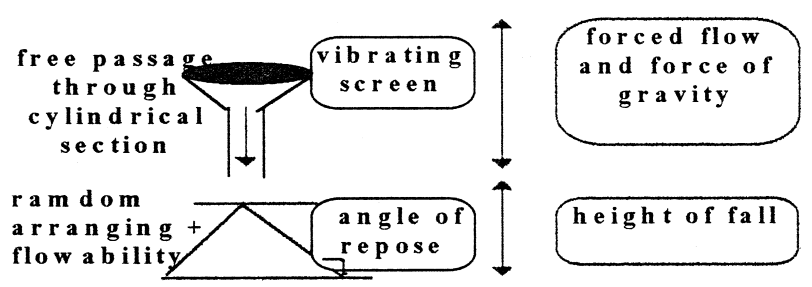

Fig. 3. Principle of measurement of angle of repose $\left(^{\circ}\right)$. 


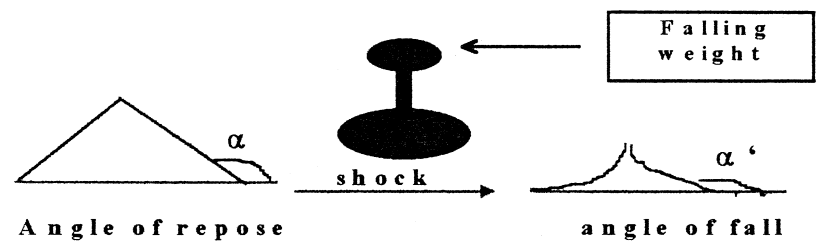

Fig. 4. Principle of measurement of angle of fall $\alpha^{\prime}$.

of repose. This angle of fall gives an indication of the floodability of the powder (Fig. 4).

\section{Flowability and floodability}

The floodability is obtained from the difference between the angle of repose $\alpha$ and of fall $\alpha^{\prime}$. The floodability shows stability or instability of a powder and so the ease with which it flows under strain $[7,8]$. The greater difference between angles of repose and of fall, the greater the instability in the behaviour of the powder. The evolution of the floodability curve varies with the nature and concentration of the surfactant as in Fig. 5.

To try to understand these complex floodability patterns, we studied the parameters influencing the floodability of powders for example:

- particle size distribution

- compressibility

-cohesiveness of the powder, obtained by the measurement of the angle of repose

-deformability of the powder under mechanical strain

(shocks), measured by the angle of fall.

\subsection{Particle size distribution}

Particle size distributions was determined using an electron beam microscope.

Without surface treatment, powders consist of different populations, each with a large particle size spread $\left(d_{90}-d_{10}\right.$ $=120 \mu \mathrm{m}$ ) as shown on photograph (Fig. 6).

Without surface treatment, co-grinding is a process of division.

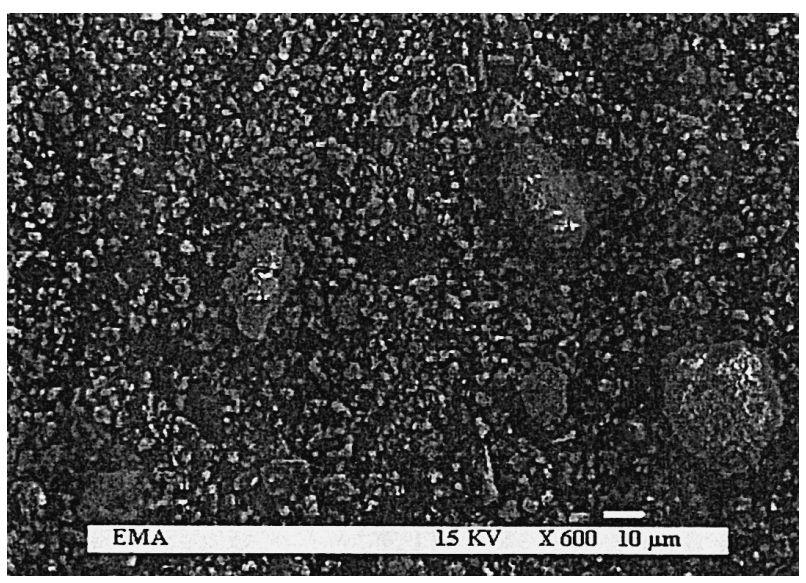

Fig. 6. Photograph taken by EBM $(\times 600)$ co-grinding without surface treatment.

With surface treatment, we observe a reduction of particle size distribution. The particle size distribution consists of two populations $D(70 \mu \mathrm{m})$ and $d(25-40$ $\mu \mathrm{m})$, but their proportions differ according to the nature of surfactant used. With surfactant A, we have a large proportion of particles $D$ while with surfactant B, a large quantity of particles $d$. The photographs of Fig. 7a and b show the effect.

With surfactant, the co-grinding is a process of agglomeration giving:

-a reduction of spread in particle size $|D-d|$

-a homogenisation of the size of particles with two populations $|D|$ and $|d|$

-a ratio of sizes $D / d$ varying with the nature of surfactant.

\subsection{Compressibility (\%)}

The apparent aerated density does not vary whatever the concentration and nature of surfactant (Figs. 8 and 9). The apparent packed density values are high percentage values of the apparent aerated density whatever the concentration and the nature of surfactant.

The height values of apparent packed density without surfactant $\left(\cong 2 \mathrm{~g} / \mathrm{cm}^{3}\right)$ are linked to a large spread of

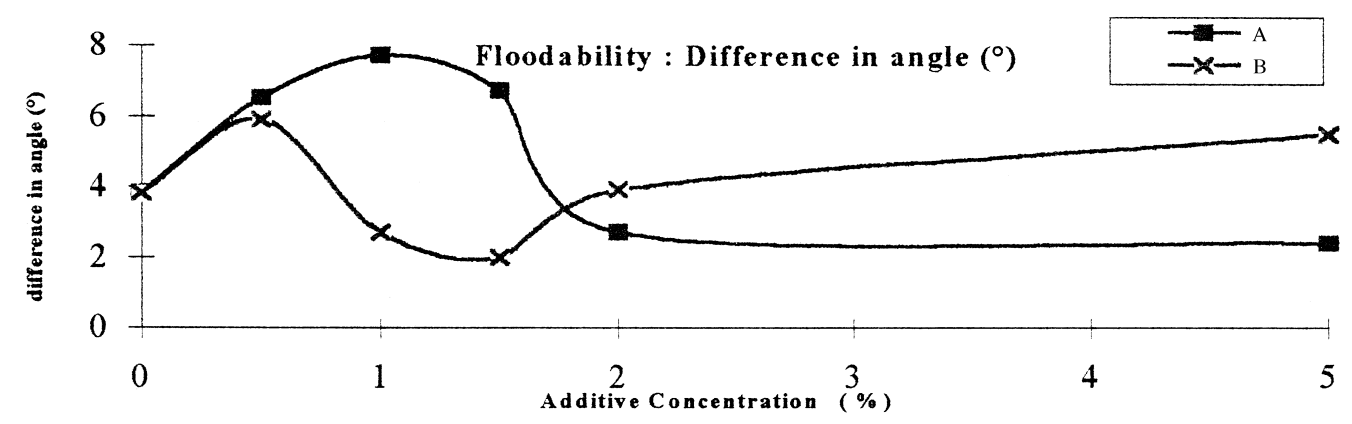

Fig. 5. Evolution of difference in angle $\alpha-\alpha^{\prime}$ as function of concentration of additive A and B. 

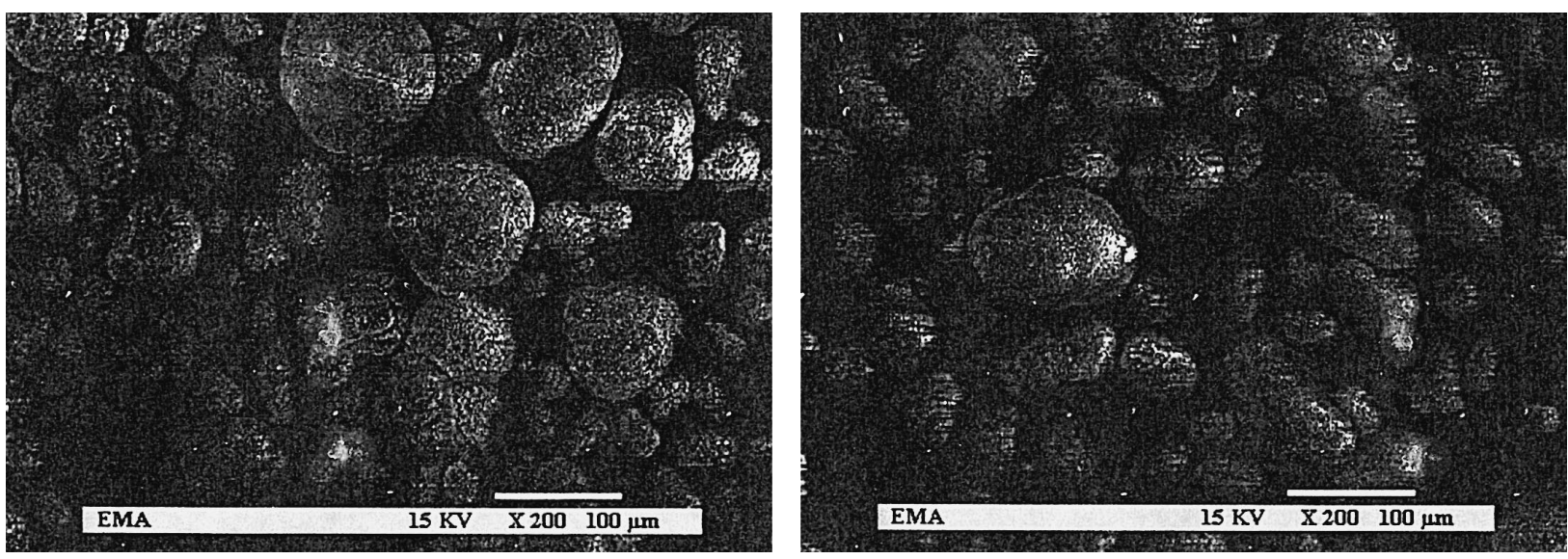

Fig. 7. (a) MEB $(\times 200)$ additive $A(2 \%)$. (b) MEB $(\times 200)$ additive $B(1.5 \%)$.

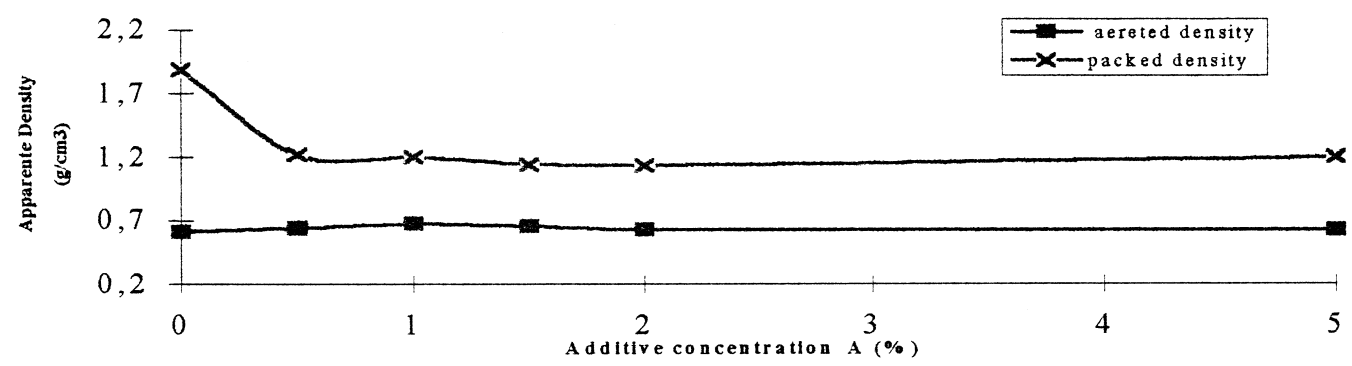

Fig. 8. Evolution of apparent packed and unpacked densities as a function of the concentration of surfactant A.

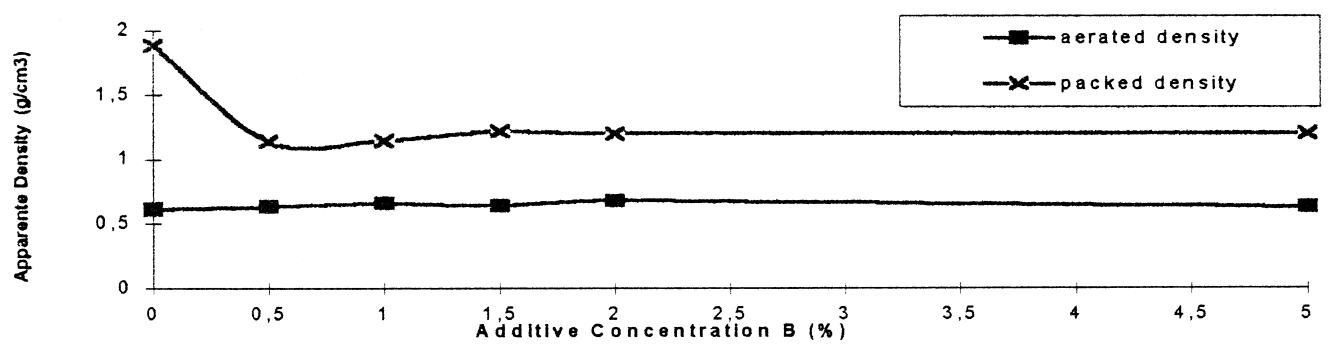

Fig. 9. Evolution of apparent packed and unpacked densities as a function of the concentration of surfactant B.

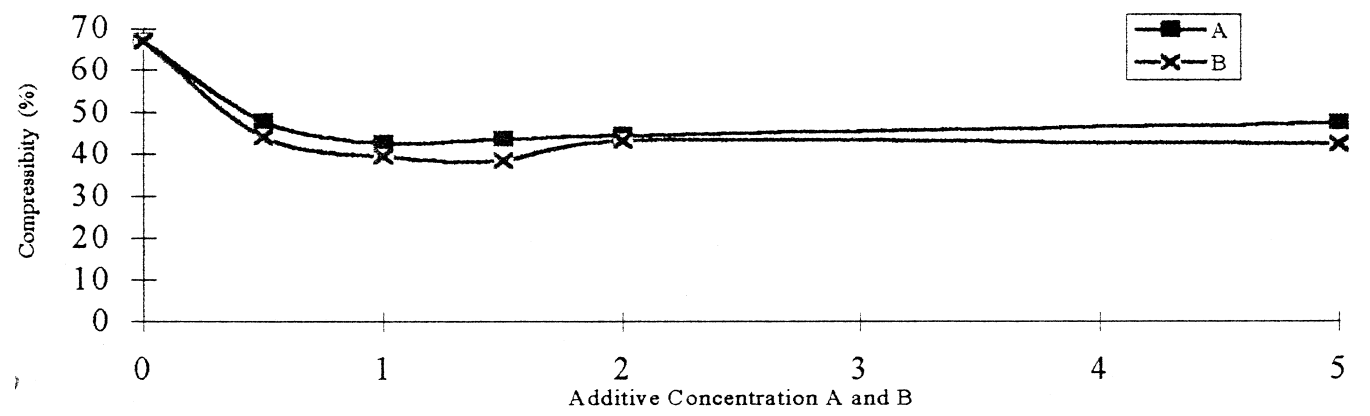

Fig. 10. Evolution of compressibility in function of concentration of surfactants A and B. 


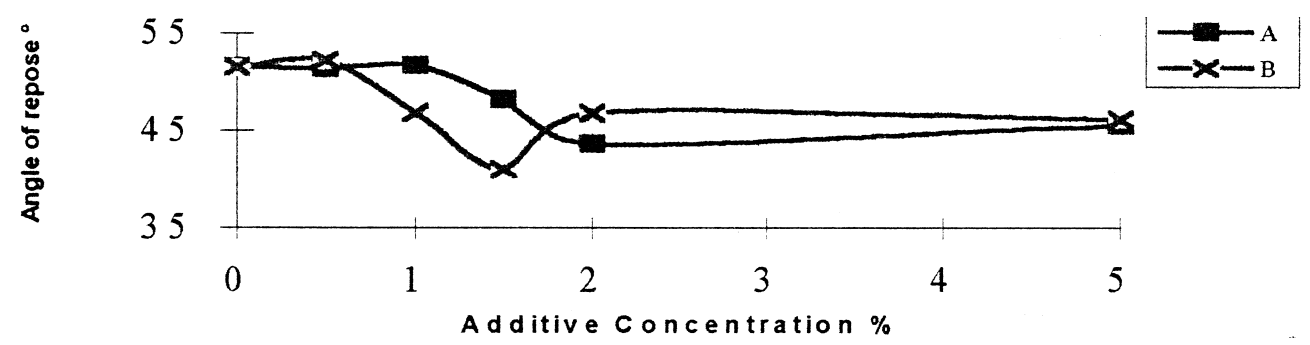

Fig. 11. Evolution of angles of repose as function of the concentration of surfactants A and B.

particle size which is a consequence of the processes of division during co-grinding.

When we add surfactants, we observe a large reduction $(50 \%)$ in apparent packed density $\left(\cong 1 \mathrm{~g} / \mathrm{cm}^{3}\right)$, the minimum corresponding to a surfactant concentration of $0.5 \%$. This reduction shows: on the one hand, the influence of surfactants both in reducing the spread in particle size obtained during co-grinding (process of agglomeration) and in reducing the strength of cohesion, linked to number of particles (which are pseudo-spherical); on the other hand, the packing of particles when we apply a mechanical strain.

Therefore, surfactants, which have polishing and lubricating effects on the surface of the particles considerably modify the apparent packed density values. Whatever the percentage and nature of surfactant A or B we obtain a non-compact compressible granular macrostructure reflecting the small spread in particle size and the existence of two populations.

The compressibility curve confirms these conclusions (Fig. 10).

Compressibility starts dropping for even small additions of surfactant: surfactant B brings it down the most (influence of the length of the carbon chain).

There exists a minimum where the compressibility is lowest when the concentration of surfactants is between 1 and $2 \%$. Beyond that, values of compressibility seem to reach a plateau but stay below initial values.

These variations are explained by the reduction of particle size distribution and a better packing of particles using surfactants.

\subsection{Study of the cohesiveness and deformability of the powder}

Measures were made three times and the error percentage is $10 \%$.

\subsubsection{Angle of repose}

We can observe two peaks on the graph in Fig. 11. (a) Values of the angle of repose were similar $\left(50^{\circ}\right)$ and constant, with a slight drop whatever the nature of the surfactant. These high values show that the powder is cohesive. (b) The two angle of repose minima correspond to surfactant content of $2 \%$ for A and 1.5 for B whatever the nature of the surfactant. We have a diminution in cohesiveness.

\subsubsection{Angle of fall}

The high values of angle of fall show the powder without surface treatment deforms itself very little under stress (Fig. 12). That confirms the cohesiveness of the powder but also a stable arrangement of the particles, due to a large spread in particle size distribution.

However, for precise values of surfactant concentration, we observe a large drop in angle of repose $(2 \%)$ by weight for surfactant A and $1.5 \%$ for surfactant B. At this level, the surfactant permits a decrease in the cohesiveness and gives greater deformability of the granular macrostructure.

The result is a particle arrangement having little stability due to a narrow particle size distribution. Beyond these, the values stay stable but below initial values.

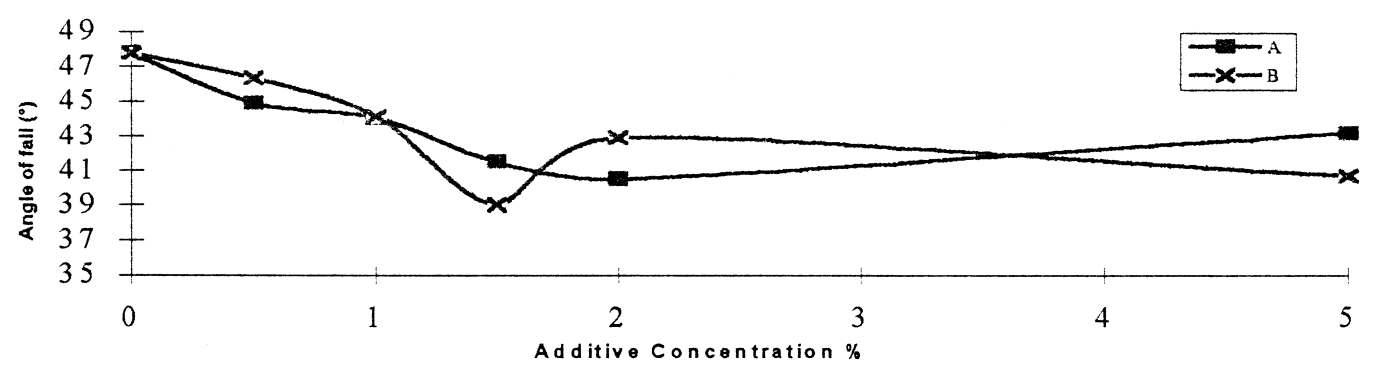

Fig. 12. Evolution of angle of fall as a function of the concentration of surfactants A and B. 


\subsection{Study of floodability}

The study of floodability is very important because this a parameter which forecasts the behaviour of the powder when it is set in motion. Floodability is a function of the difference between angle of repose and angle of fall. We can explain the evolution of the floodability curve (Fig. 5) by a complex combination of four factors: (i) Size, (ii) Compressibility, (iii) Cohesiveness, and (iv) Deformability.

These four factors are controlled by the same factors: population, size ratio, range of particle size. We can represent the system in such a way as the following.

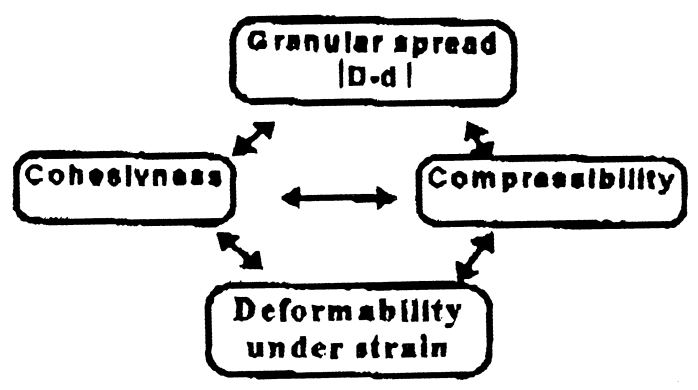

\section{Surfactant A :}

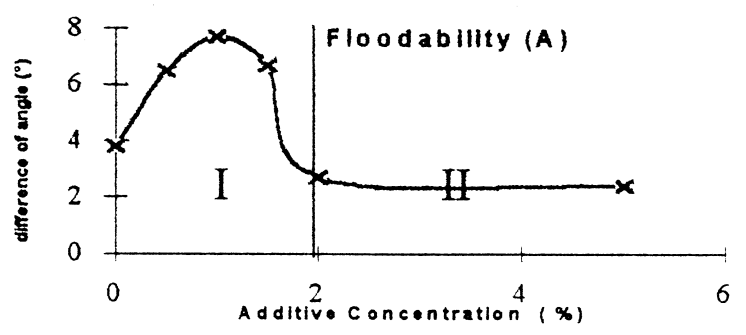

\section{Surfactant B :}

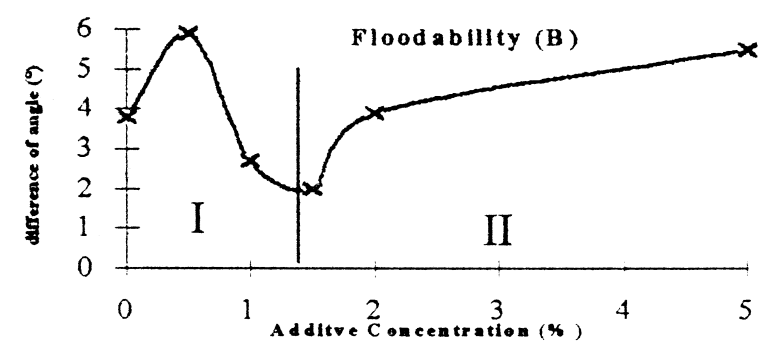

\subsubsection{Surfactant $A$}

The curve presents two distinct parts: (a) Zone I: a function of improvement in floodability [0-1.5\%], a func- tion of deterioration in floodability [1.5-2\%]; (b) Zone II: a function of floodability staying constant [2-5\%].

In zone I (concentration $<2 \%$ ), the improvement in floodability is controlled by the large drop in compressibility, due to a reduction of particle size distribution and to a reduction in cohesion. When the surfactant concentration increases, the powder becomes less and less cohesive (the angle of repose decreases) but the angle of repose does not alter under the action of strain (the angle of fall is too high). That explains a diminution of floodability in the range between $1.5 \%-2 \%$.

In zone II, surfactant concentration $>2 \%$, floodability is controlled by the cohesiveness of the powder. In effect, when surfactant concentration becomes large, there are a lot of fine particles which agglomerate (increasing cohesiveness).

\subsubsection{Surfactant $B$}

The curve evolves in more complex fashion: improving between [0-0.5\%], rising between [0.5-1.5\%], rising between 1.5 and $5 \%$.

In zone I, the evolution of floodability is the same as the one obtained with A. However, the concentration ranges in which floodability-0 to $0.5 \%$ - for the improvement and $0.5-1.5 \%$ for the drop. This difference is probably due to the length of the carbon chain.

In zone II, on the other hand, we have an increase in floodability because with surfactant B, we have a large quantity of fine particles (effect of grinding) but they do not agglomerate. In effect, the carbon chain is longer and so maintains the dispersion (sterical effect). The synergy of these different effects thus leads to a floodability optimum for a concentration between 1.5 and $2 \%$, depending on surfactant.

\section{Conclusion}

Floodability is a complex combination of compressibility cohesiveness and deformability of a powder. These factors depend on the nature and quantity of the surfactant used. This allows a reduction in spread of particle size, homogenisation of sizes and shapes (grinding and agglomeration), and a drop in cohesive strength. The result is a compromise between cohesion energy and granular macrostructure deformation-all controlled by the quantity of surfactant. The nature of surfactant also influences the rheology of the system. In effect, the length of the carbon chain plays different roles during grinding and flooding: in the former, it favours the production of large quantity of fine particles; in the latter, it can favour either agglomeration (A) or dispersion (B). The grinding/flooding function becomes complex when carbon chain length increases. 


\section{References}

[1] R.L. Carr, Chemical Engineering, 18 Jan. (1965) 163-168.

[2] W.J. Feast, H.S. Munro, Polymer Surfaces and Interfaces, Wiley, New York (1989).

[3] J. Fripiat, J. Chaussidon, A. Jelli, Chimie-physique des phénomènes de surface Masson, Paris (1971).

[4] P. Gilles de Gennes, La mécanique des poudres entre pratique et théorie, Science and Vie no. 192.

[5] L.G. Austin, R.R. Klimpel, P.T. Luckie, Process Engineering of Size
Reduction: Ball Milling, Society of Mining Engineers, New York (1984).

[6] A. Le Lan, Mélange de solides, Techniques de l'Ingénieur, Génie des Procédés, Vol. 52, Chap. A 5940 (1990).

[7] J.C. Williams, M.A. Rahman, Prediction of performance of continuous mixers for particulate solids using residence time distributions, Powder Technology, 5 (1971-1972) Part I, pp. 87-92; Part II, pp. 307-316.

[8] Jacques Duran, Sables, Poudres, et Grains, Introduction à la physique des milieux granulaires, Chap. 4, Eyrolles Paris (1997) 141-173. 